Document downloaded from:

http://hdl.handle.net/10251/105345

This paper must be cited as:

Ródenas Torralba, T.; Luz Minguez, I.; Prieto González, G.; Seoane, B.; Miro, H.; Corma Canós, A.; Kapteijn, F.... (2015). Metal organic framework nanosheets in polymer composite materials for gas separation. Nature Materials. 14(1):48-55. doi:10.1038/nmat4113

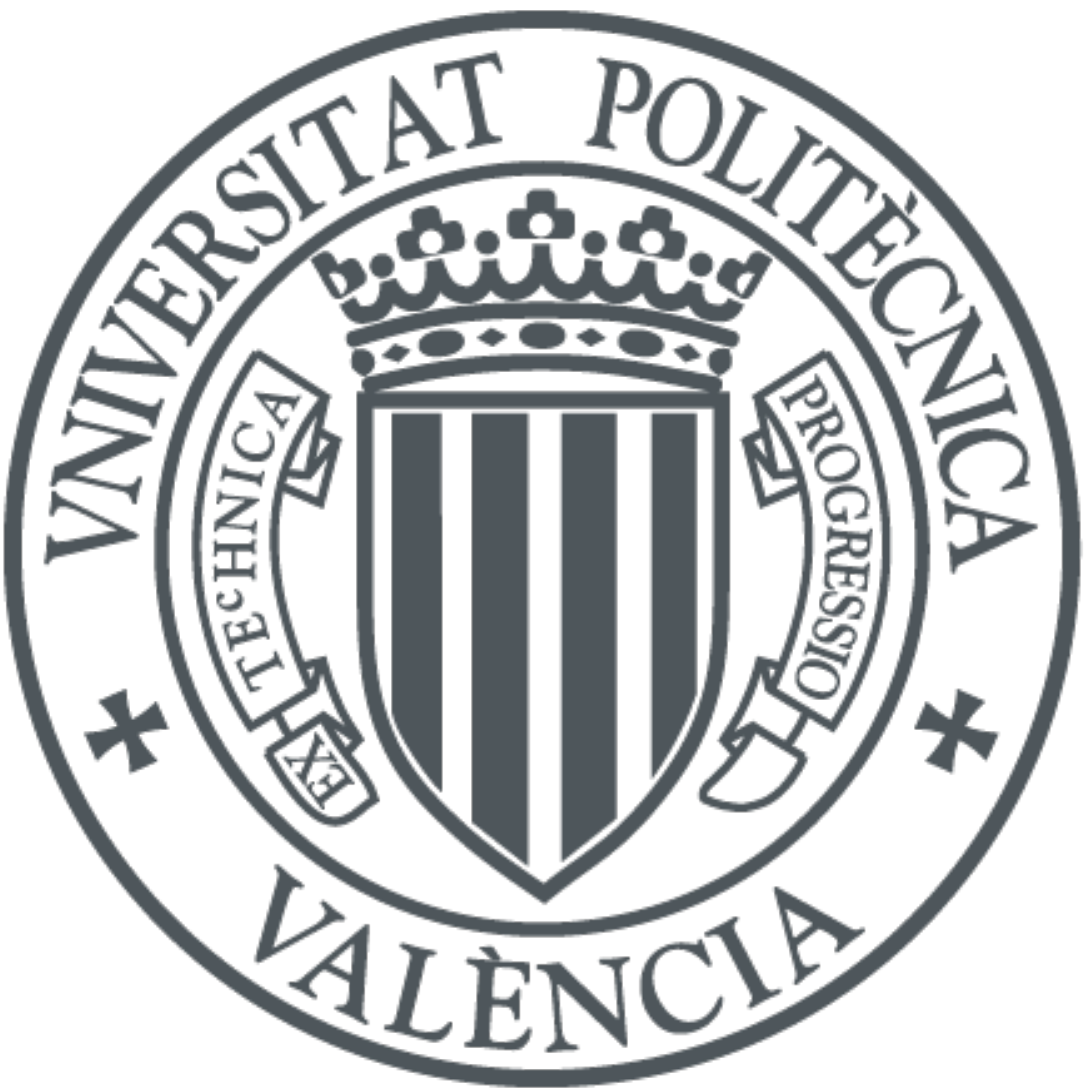

The final publication is available at

http://doi.org/10.1038/nmat4113

Copyright Nature Publishing Group

Additional Information 


\title{
Metal-organic framework nanosheets in polymer composite materials for gas separation
}

\author{
Tania Rodenas ${ }^{\# 1}$, Ignacio Luz ${ }^{\# 2}$, Gonzalo Prieto ${ }^{\# 3}$, Beatriz Seoane ${ }^{1}$, Hozanna Miro ${ }^{4}$, \\ Avelino Corma ${ }^{2}$, Freek Kapteijn ${ }^{1}$, Francesc X. Llabrés i Xamena ${ }^{2,}{ }^{,}$, and Jorge Gascon ${ }^{1,}{ }^{*}$ \\ ${ }^{1}$ Catalysis Engineering, ChemE, Delft University of Technology, Julianalaan 136, 2628 BL Delft, \\ The Netherlands ${ }^{2}$ Instituto de Tecnología Química CSIC-UPV, Universidad Politécnica de \\ Valencia, Consejo Superior de Investigaciones Científicas, Av. de los Naranjos, s/n, 46022 \\ Valencia, Spain ${ }^{3}$ Max Planck Institut für Kohlenforschung, Kaiser Wilhelm Platz 1, 45470 Mülheim \\ an der Ruhr, Germany ${ }^{4}$ Kavli Institute of Nanoscience, Delft University of Technology, \\ Lorentzweg 1, 2628 CJ Delft, The Netherlands \\ \# These authors contributed equally to this work.
}

\section{Abstract}

Composites incorporating two-dimensional nanostructures within polymeric matrices hold potential as functional components for several technologies, including gas separation.

Prospectively, employing metal-organic-frameworks (MOFs) as versatile nanofillers would notably broaden the scope of functionalities. However, synthesizing MOFs in the form of free standing nanosheets has proven challenging. We present a bottom-up synthesis strategy for dispersible copper 1,4-benzenedicarboxylate MOF lamellae of micrometer lateral dimensions and nanometer thickness. Incorporating MOF nanosheets into polymer matrices endows the resultant composites with outstanding $\mathrm{CO}_{2}$ separation performance from $\mathrm{CO}_{2} / \mathrm{CH}_{4}$ gas mixtures, together with an unusual and highly desired increment in the separation selectivity with pressure. As revealed by tomographic focused-ion-beam scanning-electron-microscopy, the unique separation behaviour stems from a superior occupation of the membrane cross-section by the MOF nanosheets as compared to isotropic crystals, which improves the efficiency of molecular discrimination and eliminates unselective permeation pathways. This approach opens the door to ultrathin MOF-polymer composites for various applications.

High-aspect-ratio nanostructured materials with extended lateral dimensions and (sub)nanometer thickness often exhibit exotic physicochemical properties radically different

\footnotetext{
Users may view, print, copy, and download text and data-mine the content in such documents, for the purposes of academic research, subject always to the full Conditions of use:http://www.nature.com/authors/editorial_policies/license.html\#terms

*Correspondence and requests for materials should be addressed to F.X.L.X. (fllabres@itq.upv.es) and J. G. (j.gascon@tudelft.nl). Author Contributions: A.C., F.K., F.X.L.X. and J.G. conceived the research. F.X.L.X. and J.G. designed the experiments and coordinated the research. I.L. synthesized and characterized the MOF materials. T.R. and B.S. synthesized and characterized the MOF-polymer composites. H.M. and T.R. recorded the FIB-SEM datasets. G.P. contributed to conception and execution of FIB-SEM data reconstruction and image analysis, with assistance of T.R. All authors contributed to analysis and discussion on the data. The manuscript was primarily written by T.R., G.P., F.X.L.X. and J.G., with input from all authors.

The authors declare no competing financial interests.
} 
from those of their isotropic (bulk) counterparts. As a result, two-dimensional nanostructures are highly interesting components for advanced structures ${ }^{[1]}$ and functional materials for applications including (opto)electronics ${ }^{[2]}$, energy storage and gas separation ${ }^{[3,4]}$. This has stimulated keen interest in devising innovative synthesis routes towards two-dimensional (2D) nanostructured materials. Essentially, these methodologies can be grouped in two categories. On one hand, top-down exfoliation approaches rely on the disintegration of three-dimensional layered solids ${ }^{[5,6,7]}$. However, shortcomings such as fragmentation, morphological damage ${ }^{[4]}$ and re-aggregation of the detached sheets are often associated with these methods. On the other hand, although scarce, bottom-up strategies which produce ultrathin materials at their genesis are preferred. In this case, 2D nanostructures might be achieved by either imposing anisotropic crystal growth ${ }^{[8]}$ or restricting thermodynamically favoured layer stacking processes ${ }^{[9]}$.

Metal-organic-frameworks are crystalline coordination polymers in which a hybrid array of metallic nodes interconnected by organic linkers defines a regular and porous structure $^{[10,11,12]}$. MOFs display pores and cavities in the range of molecular dimensions whose size, connectivity and dynamic interaction with target guest molecules can be regulated by the judicious selection of the organic and inorganic building blocks among a virtually unlimited number of possibilities ${ }^{[13,14]}$. These properties endow MOFs with an enormous application potential where molecule discrimination via preferential adsorption or molecular sieving is important, e.g. drug delivery, catalysis, and gas separation ${ }^{[15,16,17]}$. Specifically, the use of MOF-based membranes to selectively remove specific components from gas mixtures holds the promise to cause a breakthrough in several processes of economic and environmental significance ${ }^{[17,18]}$.

All-MOF membranes built upon the packing of discrete crystals ${ }^{[19]}$ face challenges which limit their applicability, e.g. complex manufacture and processing, often alongside suboptimal mechanical stability ${ }^{[20]}$. As a trade-off between the selective host-molecule interactions of MOFs and the mechano-chemical stability and easy processing of polymers, MOF-polymer composite materials have been proposed for gas separation processes ${ }^{[21,22]}$. However, conventional MOF synthesis procedures render agglomerated powders consisting of isotropic micron-sized crystals or barely dispersible nano-particles. This complicates their subsequent incorporation within a polymer matrix and restricts the integration of the two components in the ultimate composite, e.g. by promoting phase segregation. As a result, the benefits of incorporating MOF fillers within polymer matrices for gas separation applications have remained modest and manifested at relatively high MOF loadings, where the mechanical integrity of the composite is often compromised ${ }^{[23]}$. Encouraging results have been recently reported using sub-micron sized MOF filler crystals ${ }^{[2,25]}$. Intuitively, the availability of high-aspect-ratio, ideally ultrathin, MOF nanostructures would represent an advanced solution to improve the integration between both components in the composite materials, thereby circumventing the aforementioned hurdles. Bidimensional MOF structures have been manufactured on solid substrates via layer-by-layer or epitaxial growth approaches ${ }^{[18,26]}$. However, the synthesis of free-standing MOF nanosheets, which is central to intimately blend them into polymers and produce spatially uniform composites, has as yet remained a challenge. Here we present a bottom-up synthesis strategy leading to highly 
crystalline, intact MOF nanosheets that can be readily dispersed into a polymer matrix rendering composite materials with superior performance when applied as membranes in gas separation.

\section{Synthesis of free-standing MOF nanosheets}

To illustrate our synthesis approach we have selected the copper 1,4-benzenedicarboxylate (CuBDC) MOF as showcase. This material, initially synthesized by Mori et al. ${ }^{[27]}$, shows a layered crystalline structure and holds promise for the separation of polar gas molecules, such as $\mathrm{CO}_{2}$, via selective adsorption ${ }^{[28,29]}$. Its crystalline structure consists of $\mathrm{Cu}^{\mathrm{II}}$ dimers with a square-pyramidal coordination geometry interconnected by benzenedicarboxylate anions, constituting layers which stack along the $\left[\begin{array}{ll}\overline{2} & 01\end{array}\right]$ crystallographic direction (Fig. $1 \mathrm{a})^{[30]}$. The network of metal nodes and organic linkers defines nanopores which run along the stacking direction. The conventional solvothermal synthesis protocol yields predominantly well-defined cubic MOF crystals with edge dimensions ranging from 2 to 10 $\mu \mathrm{m}$ (Supplementary Fig. S1). Close-up inspection of the crystals reveals that they actually consist of multiple, closely packed lamellae (Fig. 1b).

Our bottom-up synthesis strategy to produce MOF nanosheets relies on the diffusionmediated modulation of the MOF growth kinetics. As schematically illustrated in Fig. 1c, the synthesis medium consists of three liquid layers composed of mixtures of DMF and a suitable miscible co-solvent in appropriate ratios, that are vertically arranged according to their different densities, i.e. a topmost solution of $\mathrm{Cu}\left(\mathrm{NO}_{3}\right)_{2}$ and a bottom solution of 1,4benzenedicarboxylic acid (BDCA), separated by an intermediate solvent layer. Under static conditions, diffusion of $\mathrm{Cu}^{2+}$ cations and BDCA linker precursors into the spacer segment causes a slow supply of the MOF nutrients to an intermediate region where the growth of MOF crystals occurs locally in a highly diluted medium. No immiscible liquid phases are involved in the proposed synthesis method, as opposed to interfacial reaction strategies where the extent of an organic/aqueous interface determines the surface available for MOF growth $^{[31]}$. The nascent MOF crystals are naturally removed from the reactive front by sedimentation, after which further growth is inhibited in the $\mathrm{Cu}^{2+}$-depleted underlying organic phase. X-ray diffraction of the solid product showed only three reflections which can be indexed as the $\left(\begin{array}{ll}- & 01\end{array}\right),\left(\begin{array}{ll}4 & 02\end{array}\right)$ and $\left(\begin{array}{ll}- & 04\end{array}\right)$ crystallographic planes of the CuBDC structure, all perpendicular to the stacking direction of the layers in the bulk MOF crystals and to the pore openings (Fig. 1d). None of the additional Bragg diffractions of the bulk counterpart were detected, indicative of the successful synthesis of MOF structures showing a strong preferential orientation along the basal plane. Scanning electron and atomic-force microscopies showed square lamellae displaying lateral dimensions of $0.5-4 \mu \mathrm{m}$ and thicknesses in the range of 5-25 nm, i.e. aspect ratios exceeding 20 (Fig. 1e,f and Fig. S1). Transmission electron microscopy (Supplementary Fig. S2) verified the highly regular morphology and the absence of internal structural defects in the obtained MOF nanosheets.

The versatility and scope of the MOF nanosheet synthesis strategy was further investigated by assessing the impact of relevant reaction parameters and exploring alternative MOF 
building units. Under otherwise identical synthesis conditions, omission of the intermediate buffer layer resulted in CuBDC crystals displaying notably smaller aspect ratios as a result of their up to micrometer scale thickness along the stacking direction (Supplementary Figure S3). As illustrated in Figure 2, control of the crystal growth kinetics, via adjustment of the synthesis temperature, enables variation of the thickness of the CuBDC MOF nanosheets. Upon increasing the synthesis temperature, the MOF crystal morphology evolved from ultrathin nanosheets (with average thickness $<10 \mathrm{~nm}$ ) at $298 \mathrm{~K}$ to thicker platelets at $323 \mathrm{~K}$. At a higher temperature of $333 \mathrm{~K}$, the anisotropic crystal growth was not preserved, and the synthesis yielded primarily CuBDC nanometric crystals, with aspects ratios close to unity and sizes in the range of 30-500 nm, coexisting with few bulkier crystals and platelets. The nature of the co-solvent employed serves also to modify the crystal growth behavior to obtain CuBDC crystals with different aspect ratios (Supplementary Figure S4). In addition, the same synthesis methodology can be successfully extended to produce high-aspect-ratio sheet crystals of a variety of layered MOF structures via either substitution of $\mathrm{Cu}^{2+}$ for alternative metal nodes, e.g. $\mathrm{Co}^{2+}$ and $\mathrm{Zn}^{2+}$ (Fig. 2e,f), or BDC for alternative dicarboxylate linkers (Fig. 2g,h), which represent powerful strategies to tune the MOF porosity and functionality. No surfactants or tensioactive additives are employed to modify the crystal growth pattern. Thus, application of the three-layer synthesis strategy to MOFs with propensity to more isotropic growth modes essentially preserved their crystal morphology, although modulation of the crystal growth resulted in sub-micron sized MOF crystals (Supplementary Figure S5). Collectively, these results underscore the versatility of the synthesis methodology to produce free-standing, 2D nanocrystals of several metal-organicframeworks.

Microporous MOFs containing coordinatively unsaturated (cus) copper sites, including $\mathrm{CuBDC}{ }^{[28]}$, but also related frameworks such as copper hydroxyterephthalate $(\mathrm{Cu}(\mathrm{OH}-$ $\mathrm{BDC}))^{[32]}$, and copper $1,3,5$-benzenetricaboxylate $(\mathrm{Cu}(\mathrm{BTC}))^{[33,34]}$, show potential for gas separation applications owing to their preferential $\mathrm{CO}_{2}$ adsorption over apolar molecules such as $\mathrm{CH}_{4}$ and $\mathrm{N}_{2}$. Figure 3 a shows the $\mathrm{N}_{2}$ sorption isotherms $(77 \mathrm{~K})$ for the herein synthesized bulk-type and nanosheet CuBDC MOFs.

The MOF nanosheets feature a specific surface area $\left(S_{\mathrm{BET}}\right)$ of $53 \mathrm{~m}^{2} \mathrm{~g}^{-1}$, which is mostly external, as deduced from the corresponding $t$-plot analysis, and is ca. fivefold higher than that of the bulk material $\left(S_{\mathrm{BET}} 11 \mathrm{~m}^{2} \mathrm{~g}^{-1}\right)$. Effective blockage of $\mathrm{N}_{2}$ and Ar from the microporous structure in both cases indicates that effective pore apertures smaller than the crystallographic value of $5.2 \AA{ }^{[35,36]}$ are obtained following the herein adopted synthesis and activation procedures. The $\mathrm{N}_{2}$ uptake observed at high relative pressures $\left(P / P_{0}>0.7\right)$ in the nanosheet material, featuring a H1-type hysteresis loop, and which is totally absent in the bulk solid, is characteristic of the "house-of-cards" interparticle porosity previously described for other delaminated materials ${ }^{[5,8,37,38]}$. Figure $3 \mathrm{~b}$ displays the $\mathrm{CO}_{2}$ and $\mathrm{CH}_{4}$ adsorption isotherms for CuBDC in both crystal morphologies. The isotherms show a type I shape, characteristic of microporous materials ${ }^{[39]}$, with $\mathrm{CO}_{2}$ uptakes at 1 bar (750 torr) of 1.29 and $0.82 \mathrm{mmol} \mathrm{g}^{-1}$ for $b$-CuBDC and $n s-\mathrm{CuBDC}$, respectively. The obtained values are in good agreement with previous reports on the bulk material ${ }^{[28,36]}$. The slightly higher gas uptake determined for $b$-CuBDC over $n s$-CuBDC might be ascribed to the contribution of 
inter-lamella voids to adsorption in the former. Analysis of the $\mathrm{CO}_{2}$ adsorption isotherms with the Dubinin-Radushkevich formalism ${ }^{[40]}$ revealed analogous micropore specific surface areas of 288 and $267 \mathrm{~m}^{2} \mathrm{~g}^{-1}$ for the bulk and nanosheet MOF crystals, respectively. Irrespective of the crystal morphology, the materials show a significant preference for $\mathrm{CO}_{2}$ adsorption over $\mathrm{CH}_{4}$. Ideal $\mathrm{CO}_{2} / \mathrm{CH}_{4}$ selectivities, determined as the ratio of the single component sorption capacities at every pressure, are similar for both crystal morphologies (3.9 \pm 0.5 ), comparable to those reported for related MOF structures comprising cus copper sites such as $\mathrm{Cu}(\mathrm{BTC})^{[34]}$ and $\mathrm{Cu}(\mathrm{OH}-\mathrm{BDC})^{[32]}$. The obtained results for $n s-$ and $b$-CuBDC demonstrate the similar intrinsic sorption properties of both MOF crystal morphologies and demonstrate the suitability of the $\mathrm{CuBDC}$ framework for the selective removal of $\mathrm{CO}_{2}$ from $\mathrm{CO}_{2} / \mathrm{CH}_{4}$ gas mixtures.

\section{MOF-polymer composites assembly and structure}

The structural and physicochemical properties of the CuBDC MOF nanosheets, alongside their free-standing and dispersible nature, represent promising features for their integration in advanced composites for gas separation applications. To test this potential, MOF-polymer composites were prepared by incorporating CuBDC nanosheets within a polyimide (PI) matrix at different filler loadings (2-12 wt \%). The nanosheets were dispersed in a solution of commercially available PI. Next, the composites were cast as thin membranes with a thickness of 30-50 $\mu \mathrm{m}$ (Supplementary Fig. S7) and activated under dynamic vacuum at 453 $\mathrm{K}$. The same procedure was employed to prepare comparative composites incorporating either bulk-type or sub-micron sized (nanoparticle) isotropic CuBDC MOF crystals as fillers, as well as a MOF-free polymeric film. The resulting composites are hereafter labelled as $x$-CuBDC $(y) @ \mathrm{PI}$, where $x$ is $n s, b$ or $n c$ for CuBDC nanosheet, bulk and nanoparticle crystals, respectively, and $y$ indicates the MOF weight-loading. The internal structure of the composite membranes was studied with tomographic focused-ion-beam scanning-electronmicroscopy (FIB-SEM) ${ }^{[41,42]}$ as illustrated in Fig. 4 for $n s$-CuBDC(8)@PI and $b$ CuBDC(8)@PI. A trench was carved on the upper surface of the membranes using a FIB (Fig. 4a) and a series of SEM micrographs were recorded of cross-sections exposed upon successive FIB milling of thin slices (Figs. 4b,c). After alignment of the stack of micrographs, the imaged volumes were reconstructed in 3D. The full tomograms are provided as Supplementary Videos 1 and 2, while Figs. 4e,f display surface-rendered views after segmentation of different phases. The MOF content in the examined volume was very close to the overall loading (Supplementary Methods). Despite the identical filler content, striking differences in the nanostructure were immediately evident. Whereas the regular MOF crystals leave a significant fraction of the composite volume unoccupied in $b$ CuBDC(8)@PI, due to their bulky nature, the MOF lamellae are uniformly distributed over the inspected volume for $n s-\mathrm{CuBDC}(8) @ \mathrm{PI}$.

Image analysis of the FIB-SEM tomograms allowed quantification of a number of structural parameters of the composite membranes (Fig. 5). The MOF nanosheets in $n s-$ CuBDC(8)@PI exposed ca. one order of magnitude larger surface area than the bulk-type crystals incorporated to $b$-CuBDC(8)@PI $\left(2.2 \cdot 10^{-3}\right.$ vs. $\left.2.9 \cdot 10^{-4} \mathrm{~nm}^{2} / \mathrm{nm}^{3} \mathrm{MOF}\right)$, enormously increasing their interaction with gas molecules. Of particular relevance for the separation performance is the extent to which the MOF filler occupies the membrane cross- 
section perpendicular to the gas flux, i.e. perpendicular to the pressure gradient established over the membrane during the separation process. Such flux direction is normal to the basal plane of the membranes, corresponding to the $y$-axis in the tomograms depicted in Fig. 4. Fig. 5 shows the 2D projections of the reconstructed FIB-SEM tomograms as well as the evolution of the MOF surface coverage as a function of the membrane depth along the $y$ axis.

Significant variations in the local MOF coverage at different membrane depths are observed for $b-\mathrm{CuBDC}(8) @ \mathrm{PI}$, corresponding to alternating regions with high and low MOF content due to the bulky character of the crystals. In sharp contrast, the MOF nanosheets occupy very uniformly the membrane cross-section at all depths. As a result, the effective MOF surface occupation, accumulated over an identical sampled depth of $5 \mu \mathrm{m}$, is almost three times higher, i.e. $94 \%$ versus $36 \%$, for the composite membrane incorporating CuBDC nanosheets. An intermediate case, with $51 \%$ accumulated coverage, is realized with CuBDC isotropic nanocrystals as fillers at the same MOF loading (Supplementary Figure S8). The statistical orientation of the MOF nanosheets in $n s-\mathrm{CuBDC}(8) @$ PI was also investigated. Notably deviating from a random orientation, the histogram shows a strong prevalence of lamellae oriented at angles close to $90^{\circ}$ with respect to the gas flux direction (Fig. 5e). Similar preferential orientations of nanosized two-dimensional objects within viscous matrices have been previously encountered under external shear forces ${ }^{[43]}$ as those applied during the casting of the membranes investigated here, suggesting their relevance for the ultimate nanosheet orientation in the MOF nanosheet-polymer composite films. The preferential orientation of the MOF nanosheets means that the efficiency with which the lamellae cover the membrane cross-section, exposing their pore system in the direction of the gas flux, is close to maximum (Fig. 5f), thereby minimizing the filler content and the membrane thickness required for an effective coverage. In summary, image analysis results directly prove how the incorporation of $\mathrm{CuBDC}$ nanosheets results in a notably superior occupation of the membrane cross-section perpendicular to the gas flux by the molecular sieve, increasing the likelihood of repeated molecule discrimination events and efficiently eliminating MOF-free diffusion pathways.

\section{Gas separation application}

To assess their technological relevance, the MOF-polymer composite membranes were tested in the separation of $\mathrm{CO}_{2}$ from $\mathrm{CO}_{2} / \mathrm{CH}_{4}$ mixtures. The selective recovery of $\mathrm{CO}_{2}$ from gas mixtures is central for a number of energy-related processes as well as to reduce the emissions of greenhouse gases to the atmosphere ${ }^{[44]}$. Exemplary, $\mathrm{CO}_{2}$ is a main impurity in most natural and shale gas wells. Its separation from $\mathrm{CH}_{4}$ is mandatory for the processing and transport of these carbon resources as it decreases significantly the calorific power and contributes to pipeline corrosion. While conventional amine absorption technologies are energy intensive and employ hazardous chemicals, the development of membranes for the selective separation of $\mathrm{CO}_{2}$ holds the promise for an energy-efficient and environmentally benign alternative ${ }^{[45,46]}$. As shown in Fig. 6, the incorporation of bulk-type CuBDC crystals into the polyimide matrix slightly worsens the separation selectivity as compared to a neat polyimide reference membrane. This result is illustrative of the discouraging separation performances previously encountered for composite membranes incorporating bulky, 
isotropic filler crystals ${ }^{[23]}$. It can be attributed to the disruption of the polymer chains due to the presence of the bulky filler particles, which worsens the intrinsic separation properties of the polymeric matrix and favors the generation of unselective nano- or micro-voids at the filler-matrix boundary. The use of smaller, sub-micron sized CuBDC crystals results in a slight improvement in the separation selectivity, which nevertheless underperforms the neat polymeric membrane. However, the beneficial role of the MOF nanosheets as filler material is immediately apparent. At every studied trans-membrane pressure difference, the separation selectivity for $n s-\mathrm{CuBDC}(8) @ \mathrm{PI}$ is $30-80 \%$ higher than for the polymeric membrane and $75 \%$ to 8 -fold higher than for the $b$-CuBDC(8)@PI counterpart in the range of operation conditions investigated. The similar intrinsic sorption properties of bulk-type and nanosheet CuBDC crystals cannot account for such remarkable differences in separation performance, which are therefore attributed to the different MOF crystal morphology, which is in turn key for the filler-polymer integration and the occupation of the gas permeation pathways by the molecular sieve. Most remarkably, the selectivity achieved with $n s$ $\mathrm{CuBDC}(8) @ \mathrm{PI}$ is retained or even increases slightly upon increasing the upstream pressure. This significant finding is completely opposite to the general observation for both polymeric and conventional MOF-polymer membranes ${ }^{[47]}$, that the separation selectivity drops on incrementing the partial pressure of $\mathrm{CO}_{2}$. Such classical behavior is exemplified here by the performance of the neat polymer (PI), but also composites containing isotropic filler particles such as $n c$-CuBDC(8)@PI and most notably $b$-CuBDC(8)@PI, incorporating bulkier MOF crystals. This phenomenon, which represents a notorious challenge to the state-of-the-art membranes, has been associated to the swelling of the polymer matrix upon increasing the uptake of the highly sorbing $\mathrm{CO}_{2}$, which promotes the formation of less selective pathways for the permeating gases.

The superior occupation of the membrane cross-section by the nanosheet filler, uniformly at different depth levels, has two positive effects on the separation performance. First, it results in repeated gas discrimination events, contributing to higher separation selectivity, albeit at lower gas permeabilities. This is exemplified by a relationship found for $n s-\mathrm{CuBDC}(x) @ \mathrm{PI}$ membranes between the filler cross-section coverage and the selectivity increment - and $\mathrm{CO}_{2}$ permeability decrease - with respect to the neat polymer (Supplementary Figure S9). Second and most remarkably, it effectively counteracts the undesired plasticization effect, as the depletion of MOF-free permeation pathways enables the intrinsic separation properties of the MOF nanosheets to sustain the separation performance at higher operation pressures, when the separation capacity of the polymeric matrix deteriorates. Additionally, the nearoptimal orientation of the ultrathin nanofiller permits a reduction in the MOF content and membrane thickness, resulting in incremented $\mathrm{CO}_{2}$ permeability without significant penalties in the separation selectivity (Supplementary Table S1). Preliminary results indicate that an additional approach to increase gas permeability, though at the expense of separation selectivity, is to employ nanosheets of a wider-pore MOF as filler material (Supplementary Figure S10). Overall, these results emphasize the potential technological significance of the MOF nanosheet-polymer composite materials.

Our findings underline the relevance of structuring the metal-organic-framework component in the form of high-aspect-ratio nanosheets to design advanced composite membranes, 
providing a direction to reduce the thickness required to meet a given separation performance. Next to gas separation, we envisage the versatile synthesis route and the advanced structural diagnostics strategy presented here, to enable the rational development of a variety of MOF-based and other composite materials for technological applications where integrating a functional component in ultrathin devices is essential, such as lightemitting diodes, solar-light harvesting, sensing, food packaging and functional coatings and textiles.

\section{Methods summary}

\section{Synthesis of CuBDC MOF nanosheets}

CuBDC metal-organic-framework nanosheets were synthesized in a glass test tube. A linker solution composed of $30 \mathrm{mg}$ of $\mathrm{H}_{2} \mathrm{BDC}$ dissolved in a mixture of $2 \mathrm{~mL}$ of DMF and $1 \mathrm{~mL}$ of $\mathrm{CH}_{3} \mathrm{CN}$ was poured to the tube bottom. Over this solution, a mixture of $1 \mathrm{~mL}$ of DMF and 1 $\mathrm{mL}$ of $\mathrm{CH}_{3} \mathrm{CN}$ was carefully added to prevent premature mixing of the two solutions containing the precursors. Finally, a metal precursor solution composed of $30 \mathrm{mg}$ of $\mathrm{Cu}\left(\mathrm{NO}_{3}\right)_{2} \cdot 3 \mathrm{H}_{2} \mathrm{O}$ dissolved in a mixture of $1 \mathrm{~mL}$ of DMF and $2 \mathrm{~mL}$ of $\mathrm{CH}_{3} \mathrm{CN}$ was also carefully added to the tube as the top layer. The synthesis proceeded at $313 \mathrm{~K}$ for $24 \mathrm{~h}$ in static conditions, and the resulting precipitate was collected by centrifugation and consecutively washed 3 times with DMF ( $1 \mathrm{~mL}$ each step) followed by another 3 times with $\mathrm{CHCl}_{3}$ (1 mL each step). The resulting material was left suspended in $\mathrm{CH}_{2} \mathrm{Cl}_{2}$ until the synthesis of the composite materials.

\section{Synthesis of MOF-polymer composites, thin membrane casting and gas separation experiments}

The polymer Matrimid 5218 (0.4 g), was stepwise added to a MOF suspension to obtain a final mass ratio solvent/(MOF+polymer) of 90/10. The $\mathrm{MOF} /$ polymer mass ratio was selected to achieve the desired final MOF loading in the composite materials. For the casting of membranes, the viscous suspension was poured on a flat surface and shaped as a thin film under shear forces by a doctor Blade knife. Next, the solvent was removed by evaporation, first by natural convection at room temperature for 8 hours, followed by a treatment in a vacuum oven at $453 \mathrm{~K}$ (75.01 torr) for $12 \mathrm{~h}$. Gas permeation experiments were performed with an equimolar $\mathrm{CO}_{2} / \mathrm{CH}_{4}$ mixture as feed. In all cases, separation selectivity and gas permeability values are reported after a steady operation regime was reached (Supplementary Figure S11).

\section{FIB-SEM tomography}

Focused Ion Beam-Scanning Electron Microscopy (FIB-SEM) experiments were performed in a DualBeam Strata 235 (FEI) and an AURIGA Compact (Zeiss) microscopes. Slices with nominal thickness of $52 \mathrm{~nm}$ were milled away by the FIB, operating at $30 \mathrm{kV}$ and $7 \cdot 10^{3} \mathrm{pA}$. Between 124 and 150 individual SEM micrographs of the consecutive cross-sections exposed upon milling were recorded, at magnifications of 12000-25000, with a Secondary Electron Detector operated at $5 \mathrm{kV}$. The stack of images was aligned to an external feature on the membrane surface using a cross-correlation algorithm, and a stretching operation in the $y$-direction was performed to correct the foreshortening caused by the tilt angle between 
the specimen cross-section and the SEM detector. To quantify parameters of interest from the reconstructed FIB-SEM tomograms, segmentation of the different phases, i.e. polymer matrix, MOF particles and internal voids, was performed in Avizo (FEI Visualization Sciences Group).

Further details on the experimental methods can be found in the Supplementary Information.

\section{Supplementary Material}

Refer to Web version on PubMed Central for supplementary material.

\section{Acknowledgements}

The Kavli Institute of Nanoscience (TUDelft) and the Microscopy Service of the Polytechnic University of Valencia (UPV) are acknowledged for access to their microscopy facilities and Paul Alkemade (TUDelft) and J. Luis Moya (UPV) are acknowledged for their guidance and assistance in the acquisition of FIB-SEM datasets. The research leading to these results has received funding (J.G., B.S.) from the European Research Council under the European Union's Seventh Framework Programme (FP/2007-2013) / ERC Grant Agreement n. 335746, CrystEngMOF-MMM. T.R. is grateful to TUDelft for funding. G.P. acknowledges the Alexander von Humboldt foundation for a research grant. A.C, I.L. and F.X.L.X. thank Consolider-Ingenio 2010 (project MULTICAT) and "Severo Ochoa" program for support. I.L. also thanks CSIC for a JAE doctoral grant.

\section{References}

1. Stankovich S, et al. Graphene-based composite materials. Nature. 2006; 442:282-286. [PubMed: 16855586]

2. Wang QH, Kalantar-Zadeh K, Kis A, Coleman JN, Strano MS. Electronics and optoelectronics of two-dimensional transition metal dichalcogenides. Nat Nanotechnol. 2012; 7:699-712. [PubMed: 23132225]

3. Choi S, et al. Layered silicates by swelling of AMH-3 and nanocomposite membranes. Angew. Chem. Int. Ed. Engl. 2008; 47:552-555. [PubMed: 18041016]

4. Varoon K, et al. Dispersible exfoliated zeolite nanosheets and their application as a selective membrane. Science. 2011; 334:72-75. [PubMed: 21980106]

5. Corma A, Fornes V, Pergher SB, Maesen Th. L. M. Buglass JG. Delaminated zeolite precursors as selective acidic catalysts. Nature. 1998; 396:353-356.

6. Hernandez Y, et al. High-yield production of graphene by liquid-phase exfoliation of graphite. Nat Nanotechnol. 2008; 3:563-568. [PubMed: 18772919]

7. Li P-Z, Maeda Y, Xu Q. Top-down fabrication of crystalline metal-organic framework nanosheets. Chem. Commun. 2011; 47:8436-8438.

8. Choi M, et al. Stable single-unit-cell nanosheets of zeolite MFI as active and long-lived catalysts. Nature. 2009; 461:246-249. [PubMed: 19741706]

9. Hu G, Wang N, ÓHare D, Davis J. One-step synthesis and AFM imaging of hydrophobic LDH monolayers. Chem. Commun. 2006:287-289.

10. Yamamoto K, Sakata Y, Nohara Y, Takahashi Y, Tatsumi T. Organic-inorganic hybrid zeolites containing organic frameworks. Science. 2003; 300:470-472. [PubMed: 12702872]

11. Yaghi OM, et al. Reticular synthesis and the design of new materials. Nature. 2003; 423:705-714. [PubMed: 12802325]

12. Férey G. Hybrid porous solids: past, present, future. Chem. Soc. Rev. 2008; 37:191-214. [PubMed: 18197340]

13. Gücüyener C, Bergh J, Gascon J, Kapteijn F. Ethane/ethene separation turned on its head: selective ethane adsorption on the metal-organic framework ZIF-7 through a gate-opening mechanism. J. Am. Chem. Soc. 2010; 132:17704-17706. [PubMed: 21114318] 
14. Deng $\mathrm{H}$, et al. Multiple functional groups of varying ratios in metal-organic frameworks. Science. 2010; 12:846-850. [PubMed: 20150497]

15. Khaletskaya $\mathrm{K}$, et al. Integration of porous coordination polymers and gold nanorods into coreshell mesoscopic composites toward light-induced molecular release. J. Am. Chem. Soc. 2013; 135:10998-11005. [PubMed: 23672307]

16. Corma A, Garcia H, Llabrés i Xamena FX. Engineering metal organic frameworks for heterogeneous catalysis. Chem. Rev. 2010; 110:4606-4655. [PubMed: 20359232]

17. Mueller U, et al. Metal-organic frameworks-prospective industrial applications. J. Mater. Chem. 2006; 16:626-636.

18. Gascon J, Kapteijn F. Metal-organic framework membranes-high potential, bright future? Angew. Chem. Int. Ed. Engl. 2010; 49:1530-1532. [PubMed: 20127924]

19. Li YS, et al. Controllable synthesis of metal-organic frameworks: from MOF nanorods to oriented MOF membranes. Adv. Mater. 2010; 22:3322-3326. [PubMed: 20533422]

20. Gascon J, et al. Practical approach to zeolitic membranes and coatings: state of the art, opportunities, barriers, and future perspectives. Chem. Mater. 2012; 24:2829-2844.

21. Bae T-H, et al. A high-performance gas-separation membrane containing submicrometer-sized metal-organic framework crystals. Angew. Chem. Int. Ed. Engl. 2010; 49:9863-9866. [PubMed: 21082645]

22. Zornoza B, et al. Functionalized flexible MOFs as fillers in mixed matrix membranes for highly selective separation of $\mathrm{CO}_{2}$ from $\mathrm{CH}_{4}$ at elevated pressures. Chem Commun. 2011; 47:95229524.

23. Zornoza B, Tellez C, Coronas J, Gascon J, Kapteijn F. Metal organic frameworks based mixed matrix membranes: an increasingly important field of research with a large application potential. Microp. Mesop. Mater. 2013; 166:67-78.

24. Zhang C, Dai Y, Johnson JR, Karvan O, Koros W. High performance ZIF-8/6FDA-DAM mixed matrix membrane for propylene/propane separations. J. Mem. Sci. 2012; 389:34-42.

25. Li T, Pan Y, Peinemann K-V, Lai Z. Carbon dioxide selective mixed matrix composite membrane containing ZIF-7 nano-fillers. J. Mem. Sci. 2013; 425-426:235-242.

26. Makiura R, et al. Surface nano-architecture of a metal-organic framework. Nat. Mater. 2010; 9:565-571. [PubMed: 20512155]

27. Mori W, et al. Synthesis of new adsorbent copper(II) terephthalate. Chem. Lett. 1997; 26:12191220.

28. Xin Z, Bai J, Shen Y, Pan Y. Hierarchically micro- and mesoporous coordination polymer nanostructures with high adsorption performance. Cryst. Growth Des. 2010; 10:2451-2454.

29. Adams R, Carson C, Ward J, Tannenbaum R, Koros W. Metal organic framework mixed matrix membranes for gas separations. Micropor. Mesopor. Mater. 2010; 131:13-20.

30. Carson CG, et al. Synthesis and structure characterization of copper terephthalate metal-organic framework. Eur. J. Inorg. Chem. 2009:2338-2343.

31. Ameloot R, et al. Interfacial synthesis of hollow metal-organic framework capsules demonstrating selective permeability. Nat. Chem. 2011; 3:382-387. [PubMed: 21505497]

32. Chen Z, et al. Microporous metal-organic framework with immobilized -OH functional groups within the pore surfaces for selective gas sorption. Eur. J. Inorg. Chem. 2010:3745-3749.

33. Karra JR, Walton KS. Molecular simulations and experimental studies of $\mathrm{CO}_{2}, \mathrm{CO}$, and $\mathrm{N}_{2}$ adsorption in metal-organic frameworks. J. Phys. Chem. C. 2010; 114:15735-15740.

34. Liu J, Thallapally PK, McGrail BP, Brown DR, Liu J. Progress in adsorption-based $\mathrm{CO}_{2}$ capture by metal-organic frameworks. Chem Soc. Rev. 2012; 41:2308-2322. [PubMed: 22143077]

35. Seki K, Takamizawa S, Mori W. Characterization of microporous copper(II) dicarboxylates (fumarate, terephthalate, and trans-1,4-cyclohexanedicarboxylate) by gas adsorption. Chem. Lett. 2001; 30:122-123.

36. Carson CG, et al. Structure solution from powder diffraction of copper 1,4-benzenedicarboxylate. Eur. J. Inorg. Chem. 2014:2140-2145.

37. Corma A, Diaz U, Domine ME, Fornes V. AlITQ-6 and TiITQ-6: synthesis, characterization, and catalytic activity. Angew. Chem., Int. Ed. 2000; 39:1499-1501. 
38. Corma A, Fornes V, Diaz U. ITQ-18 a new delaminated stable zeolite. Chem. Commun. 2001:2642-2643.

39. Rouquerol, F.; Rouquerol, J.; Sing, K. Adsorption by powders and porous solids. Academic Press; London: 1999.

40. Dubinin MM. The potential theory of adsorption of gases and vapors for adsorbents with energetically nonuniform surfaces. Chem. Rev. 1960; 60:235-241.

41. Uchic MD, Holzer L, Inkson BJ, Principe EL, Munroe P. Three-dimensional microstructural characterization using focused ion beam tomography. MRS Bulletin. 2007; 32:408-416.

42. Rodenas T, et al. Visualizing MOF mixed matrix membranes at the manoscale: towards structureperformance relationships in $\mathrm{CO}_{2} / \mathrm{CH}_{4}$ separation over $\mathrm{NH}_{2}-\mathrm{MIL}-53$ (Al)@ PI. Adv. Funct. Mater. 2013; 24:249-256.

43. Wang X, et al. Unusual rheological behaviour of liquid polybutadiene rubber/clay nanocomposite gels: the role of polymer-clay interaction, clay exfoliation, and clay orientation and disorientation. Macromol. 2006; 39:6653-6660.

44. Yang Y, et al. Progress in carbon dioxide separation and capture: a review. J. Environ. Sci. 2008; 20:14-27.

45. Yeo ZY, Chew TL, Zhu PW, Mohamed AR, Chai S-P. Conventional processes and membrane technology for carbon dioxide removal from natural gas: a review. J. Nat. Gas Chem. 2012; 21:282-298.

46. McKeown NB, Budd PM. Polymers of intrinsic microporosity (PIMs): organic materials for membrane separations, heterogeneous catalysis and hydrogen storage. Chem. Soc. Rev. 2006; 35:675-683. [PubMed: 16862268]

47. Vinh-Thang H, Kaliaguine S. Predictive models for mixed-matrix membrane performance: a review. Chem. Rev. 2013; 113:4980-5028. [PubMed: 23548158] 

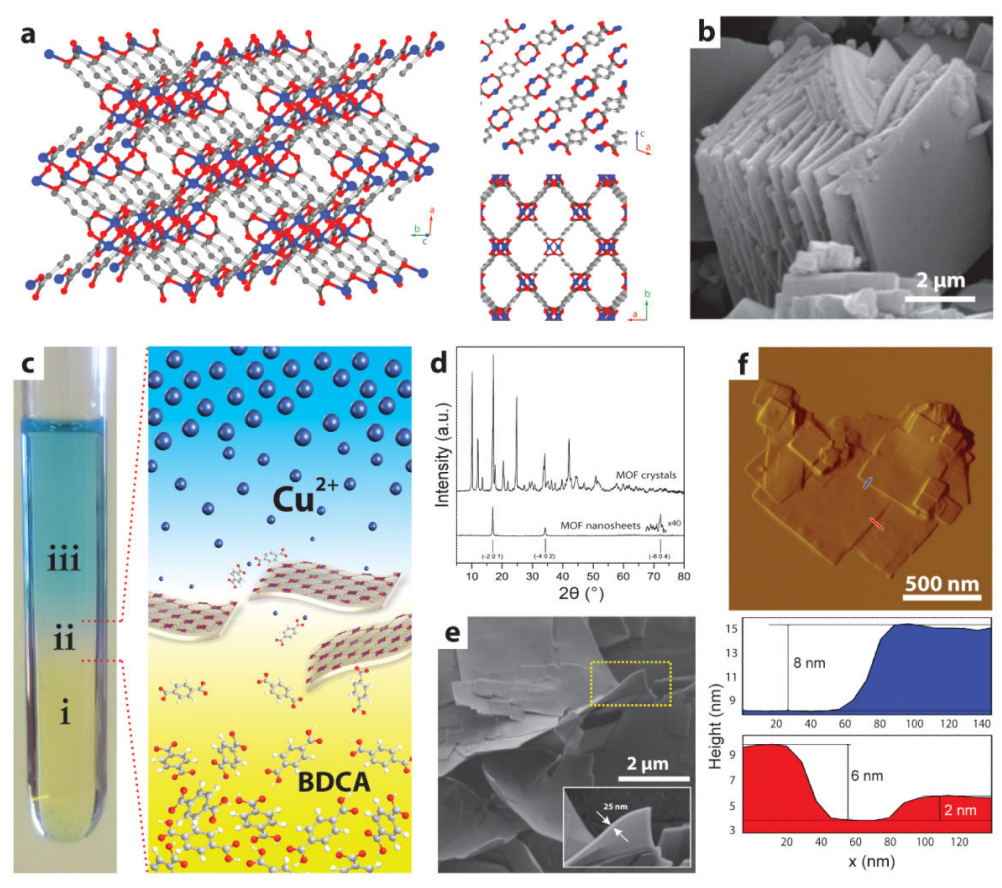

Figure 1. Synthesis and structure of metal-organic framework nanostructures a, 3D crystalline structure of CuBDC MOF. Copper, oxygen and carbon atoms are shown in blue, red and grey respectively. The insets on the right hand side display views along the $b$ (top) and c (bottom) crystallographic axis showing the stacking direction and the pore system, respectively. Hydrogen atoms and $N, N$,-dimethylformamide solvate molecules coordinated to $\mathrm{Cu}^{2+}$ ions have been omitted for clarity. $\mathbf{b}$, Scanning electron micrograph of bulk-type CuBDC MOF crystals. c, Picture showing the spatial arrangement of different liquid layers during the synthesis of CuBDC MOF nanosheets. Layers labeled as i, ii, and iii correspond to a benzene 1,4-dicarboxylic acid (BDCA) solution, the solvent spacer layer and the solution of $\mathrm{Cu}^{2+}$ ions, respectively. To enhance visualization, 2-amino 1,4benzenedicarboxylic acid, which shows a yellow color shade, has been employed as phase $\mathrm{i}$ to produce the illustrative picture presented. A close-up schematic representation of the concentration gradients established for $\mathrm{Cu}^{2+}$ and linker precursors at the spacer layer is also depicted. d, X-ray diffractograms ( $\mathrm{CuKa}$ radiation) for bulk-type and nanosheet $\mathrm{CuBDC}$ metal-organic-framework. Panels $\mathbf{e}$ and $\mathbf{f}$, show a scanning electron micrograph and an atomic-force micrograph (with corresponding height profiles), respectively, for $\mathrm{CuBDC}$ MOF nanosheets synthesized as illustrated in panel c. 

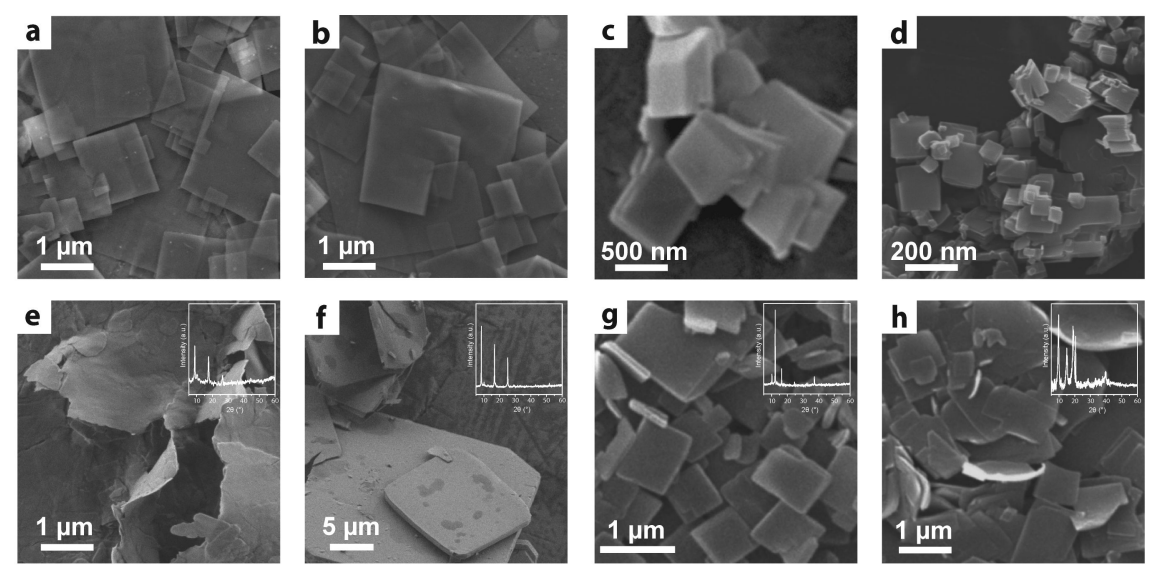

Figure 2. Versatility and scope of the three-layer synthesis strategy to produce two-dimensional MOF nanocrystals

a-d, Scanning electron microscopy (SEM) images of CuBDC crystals synthesized via the three-layer approach at a, $298 \mathrm{~K}, \mathbf{b}, 313 \mathrm{~K}, \mathbf{c}, 323 \mathrm{~K}$ and d, $333 \mathrm{~K}$. e-h, SEM micrographs of two-dimensional crystals obtained by extending the same synthesis strategy to other metalorganic-frameworks, i.e. e, cobalt 1,4-benzenedicarboxylate or CoBDC, f, zinc 1,4benzenedicarboxylate or ZnBDC, g, copper 1,4-naphthalenedicarboxylate or $\mathrm{Cu}(1,4-\mathrm{NDC})$ and $\mathbf{h}$, copper 2,6-naphthalenedicarboxylate or $\mathrm{Cu}(2,6-\mathrm{NDC})$. Insets in panels e-h display the corresponding X-ray diffractograms recorded for the 2D MOF crystals. 

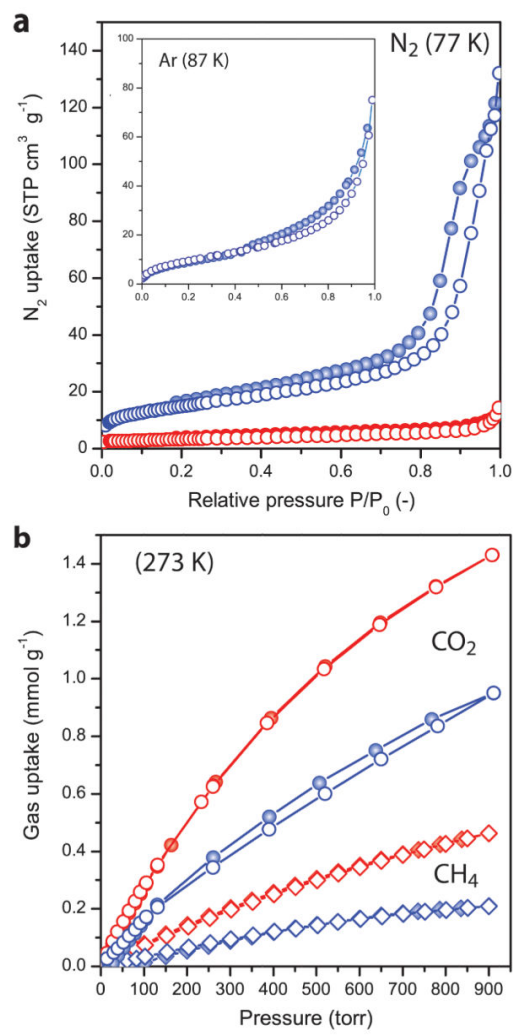

Figure 3. Sorption properties of CuBDC MOF crystals

a, $\mathrm{N}_{2}$ sorption isotherms at $77 \mathrm{~K} ; \mathbf{b}, \mathrm{CO}_{2}$ (circles) and $\mathrm{CH}_{4}$ (diamonds) sorption isotherms at $273 \mathrm{~K}$; for bulk-like (red) and nanosheet (blue) CuBDC crystals after washing and evacuation at $453 \mathrm{~K}$. The inset to panel a shows the Ar sorption isotherm at $87 \mathrm{~K}$ for the nanosheet crystals. Open symbols correspond to adsorption branches while closed symbols correspond to desorption branches. 

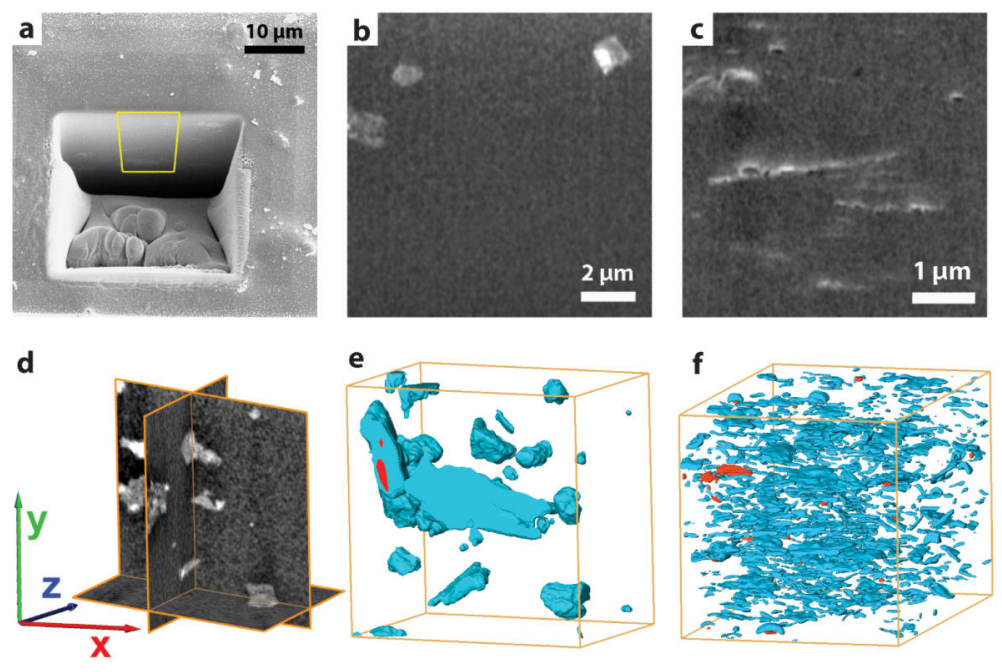

Figure 4. Tomographic FIB-SEM analysis of MOF-polymer composite membranes a, Overview scanning electron micrograph of the trench carved with a focused-ion-beam (FIB) on the surface of an $8 \mathrm{wt}$ \% MOF-polymer composite membrane. The yellow frame indicates a central region within the imaged cross-section, which was selected for further analysis. b,c, Representative SEM micrographs of cross-sections of composite membranes containing bulk-type (b) and nanosheet (c) CuBDC metal-organic-framework embedded in polyimide. MOF species appear as bright motifs on the dark grey polymer matrix. Cubic MOF crystals are perceived in panel $b$, while ultrathin MOF nanosheets are evident in panel c. d, Orthogonal cross-sections through the 3D reconstructed FIB-SEM tomogram of a MOF-polymer composite. e,f, Surface-rendered views of the segmented FIB-SEM tomograms for composite membranes containing bulk-type (e) and nanosheet (f) CuBDC metal-organic-framework embedded in polyimide. MOF particles are displayed in blue, while voids are represented in red. Given the different magnification required to image the features of interest for different composite membranes, the dimensions of the boxes shown in panels e and f along the $x: y: z$ directions are 11.2:11.2:7.6 and 4.9:4.9:6.6 $\mu \mathrm{m}$, respectively. 

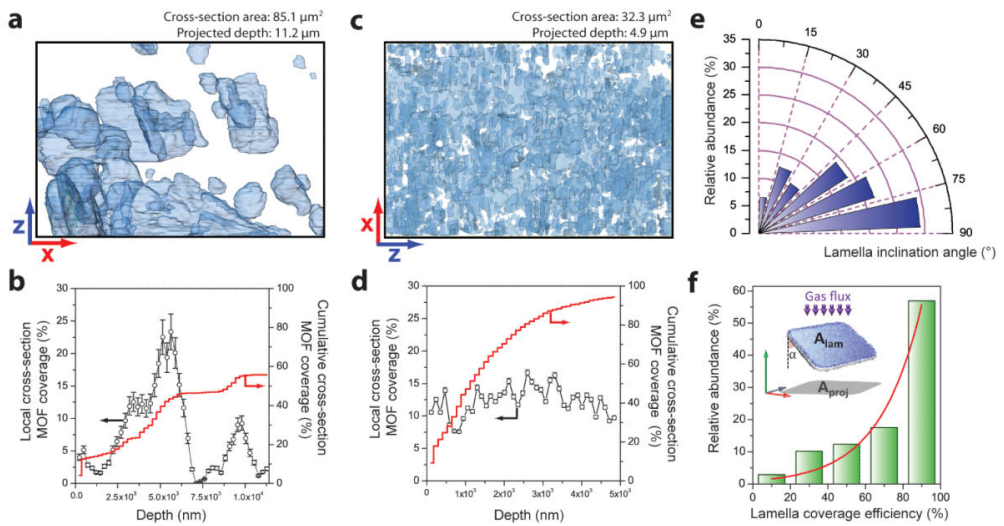

Figure 5. Image analysis of FIB-SEM tomograms for MOF-polymer composite membranes Full projections along the $y$-direction of the reconstructed volumes $(\mathbf{a}, \mathbf{c})$ and evolution of the coverage of the membrane $x z$ cross-section by MOF particles, $(\mathbf{b}, \mathbf{d})$ for composite membranes containing bulk-type (a,b) and nanosheet (c,d) CuBDC metal-organicframework embedded in polyimide. In panels a and c, the MOF particles are depicted partially transparent to better perceive overlaps in the direction of the projection. Error bars in panels $\mathrm{b}$ and $\mathrm{d}$ correspond to the standard deviation (\%). e, Angular histogram showing the orientation of MOF lamellae with respect to the gas flux direction ( $y$ axis) for a composite material containing MOF nanosheets embedded in polyimide. $\mathbf{f}$, Histogram of the efficiency with which the individual MOF nanosheets cover the membrane cross-section, defined as the ratio between the area of the MOF lamellae $\left(A_{\text {lam }}\right)$ and that projected on the plane perpendicular to the gas flux $\left(A_{\text {proj}}\right)$, as schematically depicted in the inset to the panel. In the same inset figure, a represents the angle of inclination of each MOF lamellae with respect to the $y$-axis. Green bars correspond to experimental data while the red line shows the exponential fit. See experimental methods in the Supplementary Information for more details on the tomogram image analysis procedures. 


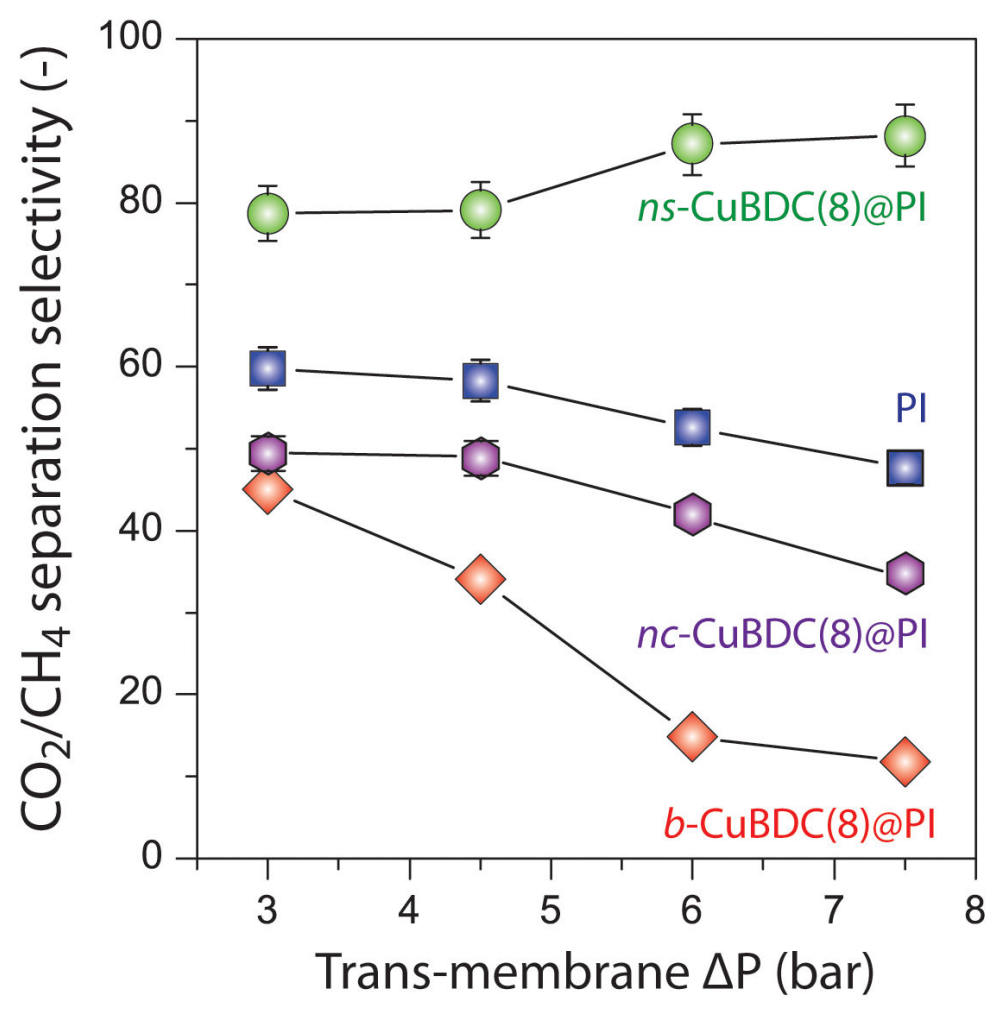

Figure 6. Application of the MOF-polymer composites in a gas separation process Separation selectivity, defined as the ratio between the permeability of $\mathrm{CO}_{2}$ and $\mathrm{CH}_{4}$, as a function of the pressure difference over the membrane for the MOF-polymer composites when employed as membranes in the separation of $\mathrm{CO}_{2}$ from an equimolar $\mathrm{CO}_{2} / \mathrm{CH}_{4}$ mixture at $298 \mathrm{~K}$. For comparison purposes, results for a neat polyimide membrane (PI) are also presented. The data correspond to steady operation conditions, after at least 8 hours on stream. $\mathrm{CO}_{2}$ permeabilities spanned in the range of 2.8-5.8 Barrer, while $\mathrm{CH}_{4}$ permeabilities were lower than 0.3 Barrer in all cases (see Supplementary Table S1). 1 Barrer $=10^{-10} \mathrm{~cm}^{3}$ (STP) $\mathrm{cm}^{-1} \mathrm{~s}^{-1} \mathrm{cmHg}^{-1}$. Error bars correspond to the standard deviations, as determined from three independent tests with selected membranes. When not displayed, error bars are smaller than the symbols. 